\title{
The glass transition and relaxation behavior of bulk water and a possible relation to confined water
}

\author{
Jan Swenson ${ }^{1, a)}$ and José Teixeira ${ }^{2}$ \\ ${ }_{1}^{1}$ Department of Applied Physics, Chalmers University of Technology, SE-412 96 Göteborg, Sweden \\ ${ }^{2}$ Laboratoire Léon Brillouin (CEA/CNRS), CE Saclay 91191 Gif-sur-Yvette Cedex, France
}

(Received 14 October 2009; accepted 14 December 2009; published online 7 January 2010)

\begin{abstract}
Due to the widespread importance of water and the difficulty to study it in the so-called no man's land between 150 and $235 \mathrm{~K}$, deeply supercooled bulk water is currently heavily debated. It speculates about its properties from extrapolations of experimental data on bulk water above $235 \mathrm{~K}$ and below $150 \mathrm{~K}$, computer simulations, and experiments on confined water for which the finite size effects may prevent crystallization in the no man's land. However, it is far from obvious how experimental data on bulk water should be extrapolated to the temperature range of the no man's land or how relevant results from computer simulations and studies of confined water are for bulk water. In this paper the structural and dynamical properties of supercooled bulk water are tried to be understood from experimental results on confined water and comparisons with bulk water. We propose that a similar crossover from a high temperature $\alpha$-relaxation to a low temperature $\beta$-relaxation occurs also for bulk water but at a higher temperature than for confined water due to the larger average number of hydrogen bonds between the water molecules in bulk water. In the case of bulk water the crossover is expected to occur around the critical temperature $\mathrm{T}_{\mathrm{s}} \approx 228 \mathrm{~K}$ when the buildup of an icelike tetrahedral network structure is completed. The proposed interpretation is the simplest one that is able to explain many of the peculiar properties of supercooled water. (C) 2010 American Institute of Physics. [doi:10.1063/1.3285286]
\end{abstract}

\section{INTRODUCTION}

It is well known that water is of central importance for life on our planet and that it has many anomalous properties compared to most liquids, such as its density maximum at $277 \mathrm{~K}$, that makes it so unique in biology. These peculiarities of water are believed to be caused by the large number of intermolecular hydrogen bonds. Since each water molecule has an ability to participate in four hydrogen bonds, a tetrahedral structure can be considered as an ideal structure, and this is the reason why ice is a network structure of these tetrahedrons. Although there is also a strong tendency of this hydrogen bonding in liquid water, it should be noted that the hydrogen bond lifetime is only about $1 \mathrm{ps}$ at room temperature, and the bonds are also substantially distorted as evident from neutron and $\mathrm{x}$-ray diffraction measurements on liquid water. $^{1-5}$

Water, as most liquids, can be supercooled below its melting temperature at $273 \mathrm{~K}$. However, unless the cooling rate is not extremely rapid, supercooled water will inevitably crystallize at about $235 \mathrm{~K}$. For very fast cooling rates $\left(>10^{5} \mathrm{~K} / \mathrm{s}\right)$, the time is insufficient for the nucleation process to occur and crystallization is avoided, but when this glassy water is reheated, it immediately crystallizes at about $150 \mathrm{~K}$. Thus, supercooled bulk water cannot be studied in the temperature range between 150 and $235 \mathrm{~K}$, which therefore is called the no man's land. If thermodynamic and dynamic properties measured above $235 \mathrm{~K}$ and below $150 \mathrm{~K}$ are ex-

\footnotetext{
${ }^{a)}$ Author to whom correspondence should be addressed. Electronic mail: jan.swenson@chalmers.se.
}

trapolated to lower and higher temperatures, respectively, problems arise since the two extrapolations are not compatible. For instance, close above $235 \mathrm{~K}$, properties such as the isothermal compressibility, ${ }^{6}$ density, ${ }^{7}$ diffusion coefficient, ${ }^{8}$ viscosity, ${ }^{9}$ and its associated main $(\alpha)$ relaxation time $\tau_{\alpha}$ (Ref. 10) follow a power-law behavior diverging at a critical temperature $\mathrm{T}_{\mathrm{s}} \approx 228 \mathrm{~K}$ (Ref. 6; see Fig. 1). On the other hand, diffusion, ${ }^{11}$ relaxation, ${ }^{12}$ and calorimetric measurements ${ }^{13}$ at temperatures below $150 \mathrm{~K}$ suggest that water exhibits a glass transition $\mathrm{T}_{\mathrm{g}}$ at about $136 \mathrm{~K}$, which, by definition, implies that the glass transition related $\alpha$-relaxation time is of the order of $100 \mathrm{~s}$ at that temperature. However, these observations obviously raise the question of how $\tau_{\alpha}$ can extrapolate to infinity at $228 \mathrm{~K}$ at the same time as it is about $100 \mathrm{~s}$ at $136 \mathrm{~K}$. To answer this question it has been proposed ${ }^{14}$ that the temperature dependence of $\tau_{\alpha}$ and other thermodynamic and transport related properties of supercooled water undergo a transition at about $228 \mathrm{~K}$ from a pronounced non-Arrhenius dependence at high temperatures to an Arrhenius behavior below the crossover temperature. Since supercooled liquids showing a highly non-Arrhenius temperature dependent viscosity are termed "fragile," in contrast to the "strong" liquids with a nearly Arrhenius dependence, ${ }^{15}$ it has thus been suggested that supercooled water exhibits a fragile-to-strong transition at about $228 \mathrm{~K}^{14}$

Since bulk water cannot be experimentally studied in the no man's land, it has become a common approach to study supercooled water in different types of geometrical confinements, such as porous glasses, zeolites, and other host materials where the water filled cavities are sufficiently small to 

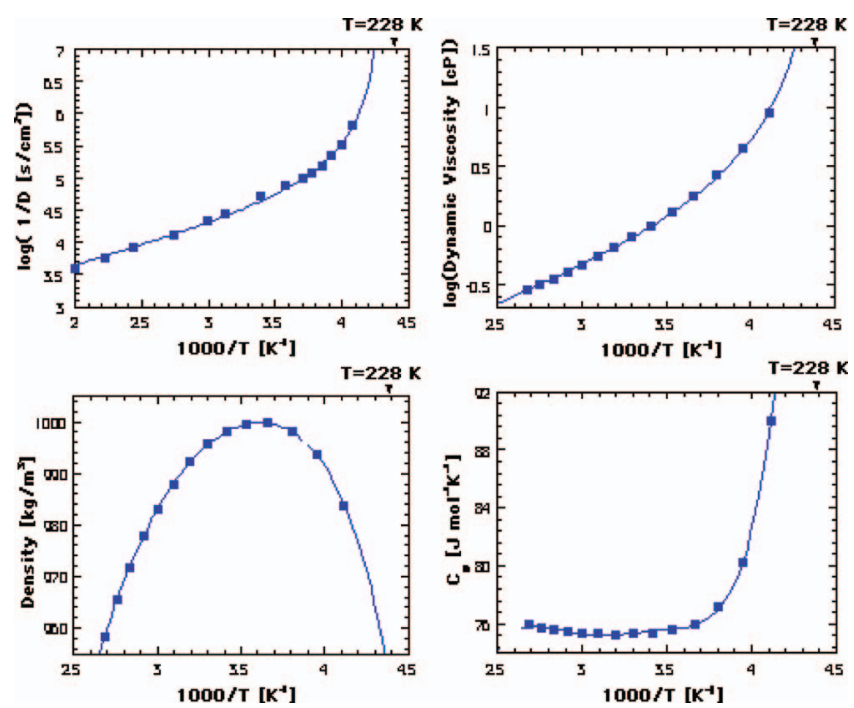

FIG. 1. Anomalous temperature dependences of water, diffusion constant (Ref. 11) (upper left panel), dynamic viscosity (upper right panel) (Refs. 46 and 47), density (lower left panel) (Ref. 48), and heat capacity $\mathrm{C}_{\mathrm{p}}$ (lower right panel) (Refs. 49 and 50). All quantities change rapidly when the critical temperature $T_{S}$ is approached from above. The lines are fits to the data points (solid squares). Power laws, diverging at $228 \mathrm{~K}$, have been used to fit the diffusion and viscosity data, whereas polynomial fits have been used to describe the density and heat capacity data.

avoid crystallization of the confined water. From studies above $235 \mathrm{~K}$, where the results can be compared with bulk water, it is clear that the structure and dynamics of confined water often are strongly altered compared to bulk water due to surface interactions as well as geometrical confinement effects. ${ }^{16-21}$ The most important reason for the structural and dynamical alterations is that the number of hydrogen bonds between the water molecules is reduced, particularly in the case of hydrophilic host materials, which also is the main reason for why crystallization is prevented. Thus, it is clear that the viscosity related $\alpha$-relaxation of water is often considerably altered in confined geometries, but it is less obvious that also local secondary $(\beta)$ relaxation processes should be equally much affected. In this paper we propose that such local dynamics in the deeply supercooled regime is relevant also for bulk water since it is determined by the relaxation of hydrogen bonds, which should not be severely affected by confinements provided that most of the water molecules interact with several other water molecules. We also suggest that the $\alpha$-relaxation of supercooled bulk water continues its temperature dependence from high temperatures without exhibiting any discontinuity or other strange behavior. With these logical and not too speculative assumptions, many of the anomalous and strange observations for bulk water can be explained.

\section{DYNAMICS OF CONFINED SUPERCOOLED WATER}

It is not our purpose to discuss the dynamical properties of confined supercooled water in detail but to mention a few anomalous observations of possible relevance also for bulk water. In Fig. 2 we show dielectric, ${ }^{20-22}$ neutron spin-echo, ${ }^{23,24}$ and ${ }^{2} \mathrm{H}$ NMR (Ref. 25) relaxation times of confined water and hydration water on proteins. From the

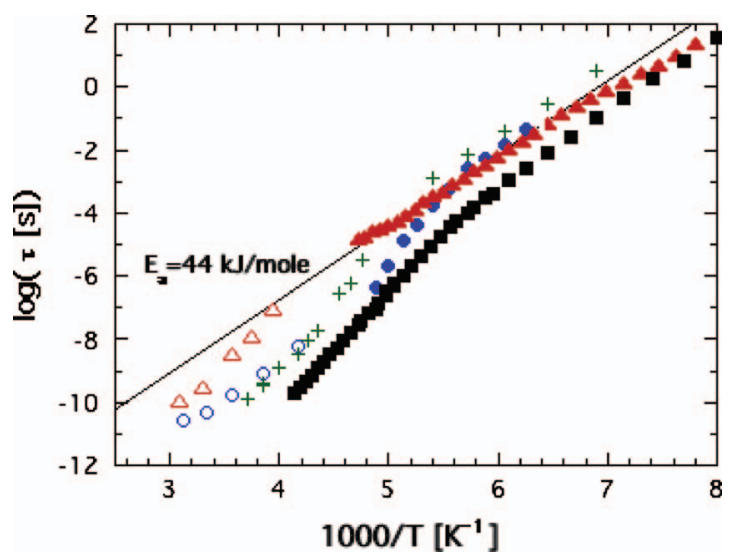

FIG. 2. Relaxations times of the main process of interfacial water in fully hydrated Na-vermiculite clay $(\triangle)$ (Refs. 20 and 23), $10 \AA$ pores of a molecular sieve (o) (Refs. 21 and 24), $21 \AA$ pores MCM-41 ( $\square$ ) (Ref. 22), and the hydrated ( $0.43 \mathrm{~g}$ water per gram of protein) protein elastin (+) (Ref. 25). Open and solid symbols are from neutron spin-echo data and dielectric relaxation data, respectively, whereas the crosses show ${ }^{2} \mathrm{H}$ NMR relaxation data (Ref. 25). Note the crossover in the temperature dependence of the relaxation process from a high temperature non-Arrhenius dependence to a low temperature Arrhenius behavior at about $190 \pm 20 \mathrm{~K}$ or when the relaxation time is approximately $10 \mu \mathrm{s}$. The line shows a typical temperature dependence of the low temperature $\beta$-relaxation. It has an activation energy of $44 \mathrm{~kJ} / \mathrm{mol}$, which also is the proposed activation energy of the corresponding process in bulk water (see Fig. 3).

figure it is clear that such water exhibits a non-Arrhenius to Arrhenius crossover in the temperature dependence of the main relaxation process that at least looks like a fragile-tostrong transition, in analogy to what has been proposed for bulk water. This crossover, occurring typically in the temperature range of $190 \pm 20 \mathrm{~K}$ [or in the case of neutron scattering data, often around $225 \mathrm{~K}$ (Refs. 26 and 27)], has mainly been thought to be either a true fragile-to-strong transition, ${ }^{26,27}$ i.e., it is the viscosity related $\alpha$-relaxation that is responsible for the relaxation process both above and below the crossover or a result of finite size effects. ${ }^{28-30}$ In the latter case it is assumed that the crossover is due to that the $\alpha$-relaxation transforms to a secondary $(\beta)$ relaxation when the cooperativity length (which grows with decreasing temperature) exceeds the size of the geometrical confinement. Indeed, in Ref. 28 it is shown that this relaxation process exhibits the expected features of a $\beta$-relaxation rather than being due to the cooperative and viscosity related $\alpha$-relaxation. For instance, (a) the relaxation peak in the frequency domain is broad and symmetric, which is the normal case of $\beta$-relaxations, but not of $\alpha$-relaxations, which are asymmetrically broadened. (b) No calorimetric glass transition is observed, in contrast to other liquids in the same (e.g., the vermiculite clay) or even more severe confinements, where the $\alpha$-relaxation is observed. ${ }^{31}$ (c) In Ref. 32 a similar relaxation process was observed for rapidly quenched bulk water by electron spin resonance measurements even in the so-called "no man's land" (150-235 K) where bulk water is mainly crystalline. This fact further supports that the observed relaxation process is of local character (in this case the relaxation of water molecules in the interface between different crystalline regions). (d) From several studies of aqueous solutions, it is clear that the relaxation process of the 
interfacial water merges with the $\alpha$-relaxation of the solution in exactly the same way as a $\beta$-relaxation merges with the $\alpha$-relaxation in deeply supercooled liquids. ${ }^{33,34}$ Thus, there are strong indications that it is a local $\beta$-like relaxation process that is the "main process" of deeply supercooled confined water and that no cooperative $\alpha$-relaxation is observable below the crossover temperature at $190 \pm 20 \mathrm{~K}$ (see Fig. 2). Similar conclusions were obtained from the ${ }^{2} \mathrm{H}$ NMR relaxation data presented in Ref. 25 , where it was stated that the low temperature water process must be a local relaxation distinguished from the cooperative $\alpha$-relaxation. These findings further imply that the dynamic crossover found at about $225 \mathrm{~K}$ in Refs. 26 and 27 can neither be due to a true fragileto-strong transition since the process they observed below $225 \mathrm{~K}$ is even faster than the process we denote as a $\beta$-relaxation, which means that it must be of even more local character.

Although it was also shown in Ref. 28 that such a confinement induced vanishing of the $\alpha$-relaxation is not unique for water, we have only observed it for other molecular liquids in the case of extremely severe confinements. For instance, the $\alpha$-relaxation of propylene glycol and its oligomers can easily be dielectrically observed even when the liquids are confined to a $0.4 \mathrm{~nm}$ thick monolayer, ${ }^{35}$ and it is equally easy to observe their calorimetric glass transitions, as mentioned above. ${ }^{31}$ This raises the question why much less severe confinements [such as cylindrical pores in MCM-41 of a diameter of $2.1 \mathrm{~nm}$ (Ref. 22)] should prevent the $\alpha$-relaxation to occur in water. A possible answer to this question is, of course, that the large number of hydrogen bonds between the molecules in water gives rise to a much larger cooperativity length of the $\alpha$-relaxation in water (particularly at low temperatures) compared to other liquids. ${ }^{36}$ However, this is an unverified assumption that leads to yet another anomalous behavior of supercooled water. Another possibility is that the crossover from a high temperature $\alpha$-relaxation to a low temperature $\beta$-relaxation is not directly caused by the confinement but is an intrinsic phenomenon in supercooled water. This behavior is then in analogy to the crossover behavior occurring for all supercooled liquids at the temperature where the $\beta$-relaxation separates from the $\alpha$-relaxation in the deeply supercooled regime. This interpretation implies that water behaves as normal liquids in confinements, with the only exception that, at least in confinements, the $\beta$-relaxation becomes considerably stronger than the $\alpha$-relaxation in the deeply supercooled regime. Support for that this is a possible scenario comes from studies of aqueous solutions, where it has been found that the dielectric intensity of the $\beta$-relaxation of the interfacial water is considerably stronger than the $\alpha$-relaxation of the solution. ${ }^{37} \mathrm{Be}$ low we will discuss the implications of these ideas for supercooled bulk water.

\section{POSSIBLE STRUCTURAL AND DYNAMICAL CHANGES OF SUPERCOOLED WATER WITH DECREASING TEMPERATURE}

Before we discuss the implications for bulk water, we have to consider the structural and dynamical alterations of water in geometrical confinements. As mentioned above, the number of hydrogen bonds between the water molecules is reduced by both surface interactions and the pure geometrical restrictions. Hence, it is not likely that a complete tetrahedral network can be built, and this prevents the confined water to crystallize and speeds up the water dynamics at low temperatures compared to bulk water, where a tetrahedral network can be formed. Since the surface interactions tend to slow down the water dynamics at high temperatures, this implies that confined water is slower than bulk water at high temperatures but faster in the deeply supercooled regime, where bulk water forms a tetrahedral structure. The situation is similar for ice where a reduced number of hydrogen bonds cause surface ice to melt at a lower temperature than bulk ice. Angell ${ }^{14}$ proposed that a tetrahedral network structure is completed in bulk water at the critical temperature $T_{\mathrm{s}}$ $\approx 228 \mathrm{~K}$ and that this is the main reason for why the water dynamics should exhibit a fragile-to-strong transition at this temperature. The structural relaxation of confined water is then expected to be faster than bulk water below $228 \mathrm{~K}$ and to show a much weaker temperature dependence around this temperature, as shown in Fig. 2. If we now assume that the observed crossover of confined water at $190 \pm 20 \mathrm{~K}$ from a high temperature $\alpha$-relaxation to a low temperature $\beta$-relaxation is an intrinsic property of supercooled water, then such a crossover is expected to occur at a higher temperature in bulk water. In fact, the most likely scenario is that the viscosity related $\alpha$-relaxation of supercooled bulk water freezes around the critical temperature of about $228 \mathrm{~K}$ when the buildup of a tetrahedral network structure is completed. This means that the $\alpha$-relaxation does not exhibit any discontinuity or other strange behavior, which most models of supercooled water are based on. Furthermore, if this amorphous network structure can be defined as a slightly disordered icelike structure, several properties of this supercooled water are expected. For instance, (a) its density should be close to that of bulk ice, (b) it should crystallize very easy, and (c) its dynamics should be icelike. (a) seems plausible from extrapolations of the measured temperature dependence of the density, ${ }^{7}$ (b) is fully consistent with the homogeneous nucleation temperature at a slightly higher temperature, and (c) is in line with dielectric relaxation studies of low-density amorphous ice, ${ }^{12}$ and the present suggestion that only local icelike relaxation processes are present in supercooled bulk water below $\mathrm{T}_{\mathrm{s}} \approx 228 \mathrm{~K}$. In fact, the timescale and activation energy of the low temperature $\beta$-relaxation in confined water (see Fig. 2) are very similar to what have been observed for hexagonal ice below $170 \mathrm{~K}^{38}$ In further support for the structural and dynamical similarities with ice in the deeply supercooled regime is the remarkably low entropy of water at $150 \mathrm{~K} .{ }^{39}$ It should here be noted that these ideas for the structure and dynamics of supercooled bulk water are not entirely new. In 2006 Teixeira et $a l{ }^{40}$ proposed a model based on quasielastic neutron scattering data, where the low temperature dynamics of bulk water is determined by the hydrogen bond relaxation, which should be considered as a local intermolecular $\beta$-like relaxation process. Teixeira et al. $^{40}$ also pointed out the importance of the formation of a tetrahedral network structure with the same symmetry that characterizes hexagonal ice. It causes crystal- 
lization at the homogenous nucleation temperature and makes the shear viscosity approaching infinity when this network structure is (almost) completed. Thus, as in ice, local relaxation processes and associated diffusion processes take place below $T_{s}$, but the water does not flow because its viscosity is basically infinitely high below this temperature. If these ideas are correct, this also implies that the true glass transition of bulk water should be close to $228 \mathrm{~K}$ and that the calorimetrically observed feature at $136 \mathrm{~K}$ (Ref. 13) should be associated with a freezing in of a $\beta$-relaxation rather than being considered as a true glass transition. In favor of this interpretation is also the small step in the heat capacity [only $2 \%$ of the $\Delta \mathrm{C}_{\mathrm{p}}$ values for $\left(\mathrm{H}_{2} \mathrm{O}\right)_{2}-\mathrm{H}_{2} \mathrm{O}_{2}$ and $\mathrm{H}_{2} \mathrm{O}-\mathrm{N}_{2} \mathrm{H}_{4}$ (Ref. 41)] at $136 \mathrm{~K}$ (Ref. 13) and the fact that this $\beta$-relaxation freezes in (or more precise, reaching a dielectric relaxation time of $100 \mathrm{~s}$ ) at about the same temperature for confined water. ${ }^{42}$ Provided that most of the water molecules in a confined geometry are hydrogen bonded to several other water molecules, such a local $\beta$-relaxation, determined by the hydrogen bond relaxation, ${ }^{40}$ should not be substantially affected by the confinement, and the results should therefore be of relevance also for deeply supercooled bulk water. Furthermore, the typical activation energy of the $\beta$-relaxation in confined water is $44 \mathrm{~kJ} / \mathrm{mol}^{42}$ which is almost identical to the activation energy of $45 \mathrm{~kJ} / \mathrm{mol}$ for diffusion in glassy bulk water, ${ }^{43}$ suggesting that this $\beta$-relaxation is also responsible for the diffusion in deeply supercooled water.

In Ref. 41 Angell compared the glass transition behavior of water with that of "glassy crystals" such as the rotor phase of the fullerene $\mathrm{C}_{60}$ and pointed out the similarities. This $\mathrm{C}_{60}$ fullerene exhibits a glass-transition-like change in $\mathrm{C}_{\mathrm{p}}$ at $90 \mathrm{~K}$ when some disorder is frozen in below that temperature. ${ }^{44}$ The step in $\mathrm{C}_{\mathrm{p}}$ is, as expected, even smaller than the step at $136 \mathrm{~K}$ for water. By definition, a glassy crystal does not show any structural $\alpha$-relaxation process due to the fact that its viscosity can be regarded as infinitely high, and therefore it is in our opinion misleading to talk about "glass transition" in the case of glassy crystals. Particularly, when comparisons to true glass-forming liquids are made, the glass transition of a glassy crystal should rather be compared with the freezing in of local secondary relaxation processes, which occur in the glassy state of glass-forming liquids, far below the true glass transition temperature. Thus, provided that an ordinary glassforming liquid should be considered to exhibit only one glass transition (i.e., when its structural $\alpha$-relaxation freezes in) and not additional glass transition(s) when its $\beta$-relaxation(s) freezes in, it is obvious that the calorimetric feature of water at $136 \mathrm{~K}$ (Ref. 13) should not be regarded as a true glass transition if it shows any similarities to the glass transition behavior of $\mathrm{C}_{60}$. The similarity to the calorimetric behavior of $\mathrm{C}_{60}$ provides, however, additional support for that the relaxation process observed in deeply supercooled water $(<150 \mathrm{~K})$ should be regarded as a $\beta$-relaxation in glassy water, as proposed in this paper and by Teixeira et al. ${ }^{40}$

With the model proposed here there is no need for a liquid-liquid transition close to $228 \mathrm{~K}$ because the experimentally observed decrease in the density of bulk ${ }^{7}$ and confined water ${ }^{45}$ in the temperature range from 277 to $225 \mathrm{~K}$ (the lower temperatures only for confined water) is precisely

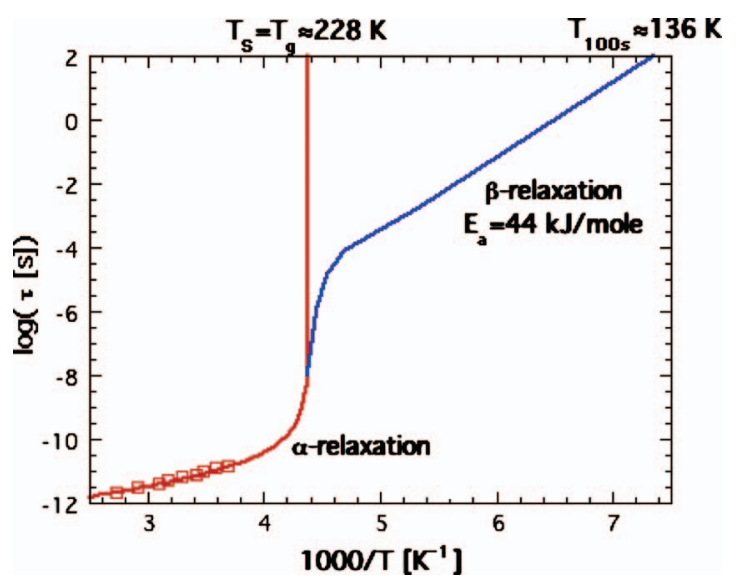

FIG. 3. A possible relaxation scenario for supercooled water with the dielectric relaxation times measured by Rønne et al. (Ref. 10) included. The relaxation time of the viscosity related $\alpha$-relaxation goes rapidly to infinity around the critical temperature of $228 \mathrm{~K}$. Below this temperature the slowest relaxation process is the more local $\beta$-relaxation, which behaves as the $\beta$-relaxation in confined water and reaches a relaxation time of $100 \mathrm{~s}$ at $136 \mathrm{~K}$. Thus, below $228 \mathrm{~K}$ water is macroscopically a solid, although relaxation and diffusion processes can still take place, as in ice.

what is expected when an icelike tetrahedral network structure is built. The density should decrease continuously from the density maximum at $277 \mathrm{~K}$ to the temperature where the tetrahedral network structure is completed, and no substantial changes in the average structure can occur. Below this temperature the density should increase again with decreasing temperature as for ice and most other solid materials.

A density minimum at the here proposed $\mathrm{T}_{\mathrm{g}}$ around $228 \mathrm{~K}$ is, of course, an anomalous behavior of a "normal" liquid. However, water, as we all know, is not a normal liquid in all aspects, and in the case of water a density minimum at $\mathrm{T}_{\mathrm{g}}$ is basically unavoidable since the density of ice is so much lower than for water and the local structure of glassy water is similar to that of ice. Thus, whenever the crystalline state of a material has a lower density than its liquid state, a density minimum around the glass transition temperature should be expected.

The rapid increase in $C_{p}$ when the temperature is approaching $228 \mathrm{~K}$ from above, as shown in Fig. 1, is, of course, an anomalous behavior for a calorimetric glass transition. It is more of a typical behavior close to a critical point. However, in the case of water the decreasing temperature gives rise to a rapid buildup of a tetrahedral network structure close to $228 \mathrm{~K}$, which causes the cooperativity length and thereby also the relaxation time of the $\alpha$-relaxation to increase equally rapid. The rapid increase in the cooperativity length can then be associated with a critical point, whereas the rapid increase in the structural relaxation time (and related viscosity) causes water to undergo a glass transition. Thus, in the case of water the glass transition temperature can also be regarded as a critical temperature.

The relaxation scenario of supercooled water we obtain from the discussion above is illustrated in Fig. 3. The cooperativity length and relaxation time of the viscosity related $\alpha$-relaxation approach infinity at the critical and glass transition temperature of about $228 \mathrm{~K}$. Below this temperature supercooled water behaves macroscopically as a solid, and 
the main (i.e., slowest) relaxation process is given by the more local $\beta$-relaxation. Since this $\beta$-relaxation, determined by the hydrogen bond dynamics, is of local character and therefore similar in different types of confinements, its properties should be close to what is typically obtained for confined water. ${ }^{42}$ This means that it has an activation energy of about $44 \mathrm{~kJ} / \mathrm{mol}$ and reaches a relaxation time of $100 \mathrm{~s}$ at $136 \mathrm{~K}$. Slightly above the glass transition temperature, the low temperature $\beta$-relaxation merges with the viscosity related $\alpha$-relaxation and follows its non-Arrhenius high temperature dependence.

\section{CONCLUDING REMARKS}

In this paper we propose a new interpretation for the structural and dynamical behavior of supercooled water. We consider it to be one of the simplest and least speculative models that are consistent with the available experimental data on water. We hope that the proposed interpretation will make the scientific community in this and water related research fields open for the suggestion that bulk water may be in its glassy state only a few kelvins into the no man's land and that it will stimulate future studies on this topic. In the model we assume that the observations for confined water, such as the dynamical crossover from a cooperative $\alpha$-like relaxation process at high temperatures to a local $\beta$-relaxation in the deeply supercooled regime, are relevant but quantitatively different for bulk water. This assumption then leads to that the most likely glass transition temperature of bulk water is close to the critical temperature $\mathrm{T}_{\mathrm{s}}$ $\approx 228 \mathrm{~K}$

\section{ACKNOWLEDGMENTS}

J.S. thanks the Swedish Foundation for Strategic Research and the Swedish Energy Agency for financial support.

${ }^{1}$ A. K. Soper and M. G. Phillips, Chem. Phys. 107, 47 (1986).

${ }^{2}$ G. Hura, J. M. Sorenson, R. M. Glaeser, and T. Head-Gordon, J. Chem. Phys. 113, 9140 (2000).

${ }^{3}$ J. M. Sorenson, G. Hura, R. M. Glaeser, and T. Head-Gordon, J. Chem. Phys. 113, 9149 (2000).

${ }^{4}$ A. H. Narten and H. A. Levy, J. Chem. Phys. 55, 2263 (1971)

${ }^{5}$ M. C. Bellissent-Funel, L. Bosio, and J. Teixeira, J. Phys.: Condens. Matter 3, 4065 (1991).

${ }^{6}$ R. J. Speedy and C. A. Angell, J. Chem. Phys. 65, 851 (1976).

${ }^{7}$ D. H. Rasmussen and A. P. MacKenzie, J. Chem. Phys. 59, 5003 (1973).

${ }^{8}$ K. T. Gillen, D. C. Douglass, and M. J. R. Hoch, J. Chem. Phys. 57, 5117 (1972).

${ }^{9}$ J. Hallett, Proc. Phys. Soc. London 82, 1046 (1963).

${ }^{10}$ C. Rønne, P. O. Åstrand, and R. S. Keiding, Phys. Rev. Lett. 82, 2888 (1999)

${ }^{11}$ R. S. Smith and B. D. Kay, Nature (London) 398, 302 (1999).

${ }^{12}$ O. Andersson, Phys. Rev. Lett. 98, 057602 (2007)

${ }^{13}$ G. P. Johari, A. Hallbrucker and E. Mayer, Nature (London) 330, 552 (1987).

${ }^{14}$ K. Ito, C. T. Moynihan, and C. A. Angell, Nature (London) 398, 492 (1999).

${ }^{15}$ C. A. Angell, J. Non-Cryst. Solids 131-133, 13 (1991).
${ }^{16}$ J. M. Zanotti, M. C. Bellissent-Funel, and S. H. Chen, Phys. Rev. E 59, 3084 (1999).

${ }^{17}$ F. Bruni, M. A. Ricci, and A. K. Soper, J. Chem. Phys. 109, 1478 (1998); A. K. Soper, F. Bruni, and M. A. Ricci, ibid. 109, 1486 (1998).

${ }^{18}$ V. Crupi, D. Majolino, P. Migliardo, and V. Venuti, J. Phys. Chem. A 104, 11000 (2000).

${ }^{19}$ S. Takahara, M. Nakano, S. Kittaka, Y. Kuroda, T. Mori, H. Hamano, and T. Yamaguchi, J. Phys. Chem. B 103, 5814 (1999).

${ }^{20}$ R. Bergman and J. Swenson, Nature (London) 403, 283 (2000).

${ }^{21}$ H. Jansson and J. Swenson, Eur. Phys. J. E 12, 51 (2003).

${ }^{22}$ J. Sjöström, J. Swenson, R. Bergman, and S. Kittaka, J. Chem. Phys. 128, 154503 (2008).

${ }^{23}$ J. Swenson, R. Bergman, and S. Longeville, J. Chem. Phys. 115, 11299 (2001).

${ }^{24}$ J. Swenson, H. Jansson, W. S. Howells, and S. Longeville, J. Chem. Phys. 122, 084505 (2005).

${ }^{25}$ M. Vogel, Phys. Rev. Lett. 101, 225701 (2008).

${ }^{26}$ A. Faraone, L. Liu, C.-Y. Mou, C.-W. Yen, and S.-H. Chen, J. Chem. Phys. 121, 10843 (2004).

${ }^{27}$ L. Liu, S.-H. Chen, A. Faraone, C.-W. Yen, and C.-Y. Mou, Phys. Rev. Lett. 95, 117802 (2005).

${ }^{28}$ J. Swenson, H. Jansson, and R. Bergman, Phys. Rev. Lett. 96, 247802 (2006).

${ }^{29}$ J. Swenson, Phys. Rev. Lett. 97, 189801 (2006)

${ }^{30}$ S. Cerveny, J. Colmenero, and A. Alegria, Phys. Rev. Lett. 97, 189802 (2006)

${ }^{31}$ S. Cerveny, J. Mattsson, J. Swenson, and R. Bergman, J. Phys. Chem. B 108, 11596 (2004).

${ }^{32}$ S. N. Bhat, A. Sharma, and S. V. Bhat, Phys. Rev. Lett. 95, 235702 (2005).

${ }^{33}$ S. Cerveny, G. A. Schwartz, A. Alegría, R. Bergman, and J. Swenson, J. Phys. Chem. B 124, 194501 (2006).

${ }^{34}$ J. Sjöström, J. Mattsson, R. Bergman, and J. Swenson, "Dielectric secondary relaxation of water in aqueous binary glass-transformers," Phys. Chem. Chem. Phys. (submitted).

${ }^{35}$ J. Swenson, G. A. Schwartz, R. Bergman, and W. S. Howells, Eur. Phys. J. E 12, 179 (2003).

${ }^{36}$ H. E. Stanley and J. Teixeira, J. Chem. Phys. 73, 3404 (1980).

${ }^{37}$ H. Jansson, R. Bergman and J. Swenson, "Role of solvent for the dynamics and the glass transition of proteins," J. Chem. Phys. (submitted).

${ }^{38}$ G. P. Johari and S. J. Jones, Proc. R. Soc. London, Ser. A 349, 467 (1976).

${ }^{39}$ R. J. Speedy, P. G. Debenedetti, R. S. Smith, C. Huang, and B. D. Kay, J. Chem. Phys. 105, 240 (1996).

${ }^{40}$ J. Teixeira, A. Luzar, and S. Longeville, J. Phys.: Condens. Matter 18, S2353 (2006).

${ }^{41}$ C. A. Angell, Science 319, 582 (2008).

${ }^{42}$ S. Cerveny, G. A. Schwartz, R. Bergman, and J. Swenson, Phys. Rev. Lett. 93, 245702 (2004).

${ }^{43}$ B. D. Kay, P. Ayotte, J. L. Daschbach, Z. Dohnalek, G. A. Kimmel, R. S. Smith, and G. R. Teeter, Abstr. Pap. - Am. Chem. Soc. 229, U706-U706 (2005)

${ }^{44}$ T. Matsuo, H. Suga, W. I. F. David, R. M. Ibberson, P. Bernier, A. Zahab, C. Fabre, A. Rassat, and A. Dworkin, Solid State Commun. 83, 711 (1992).

${ }^{45}$ D. Z. Liu, Y. Zhang, Y. Liu, L. J. Wu, C.-C. Chen, C.-Y. Moy, and S.-H. Chen, J. Phys. Chem. B 112, 4309 (2008).

${ }^{46}$ C. H. Cho, J. Urquidi, and G. Wilse Robinson, J. Chem. Phys. 111, 10171 (1999).

${ }^{47}$ C. H. Cho, J. Urquidi, S. Singh, and G. Wilse Robinson, J. Phys. Chem. B 103, 1991 (1999).

${ }^{48}$ CRC Handbook of Chemistry and Physics, 80th ed., edited by D. R. Lide (CRC, Boca Raton, 1999).

${ }^{49}$ G. P. Johari, A. Hallbrucker, and E. Mayer, Science 273, 90 (1996).

${ }^{50}$ National Institute of Standards and Technology, A gateway to the data collections, available at http://webbook.nist.gov. 